\title{
One-step size-controlled synthesis of functional graphene oxide/silver nanocomposites at room temperature.
}

\begin{abstract}
A stable aqueous suspension of functional graphene oxide/silver nanocomposite (FGO/Ag) was prepared in an alkaline medium by a simple room temperature stirring method. Functional graphene oxide (FGO) served as substrate, reducing agent and stabilizer for the silver nanoparticles (Ag-NPs). The $\mathrm{pH}$ of the solution played a prominent role in the formation of the Ag-NPs. The morphology of the nanoparticles (NPs) could be controlled by adjusting the $\mathrm{pH}$ between 9.51 and 12.62 by adding $\mathrm{NaOH}$ solution. The aqueous stability of the nanocomposites was greatly improved by the attached functional groups. UV-visible spectroscopy, transmission electron microscopy (TEM) and field emission scanning electron microscopy (FESEM) images suggested the formation of spherical, Ag-NPs with a narrow size distribution at $\mathrm{pH} \mathrm{11.40.} \mathrm{The}$ nanocomposites showed high bactericidal activity against Escherichia coli bacteria and also enhancement in Raman intensity due to surface enhanced Raman scattering (SERS), which was found to be dependent on the size distribution of the Ag-NPs. This work provides a simple, scalable and environmentally friendly approach to the preparation of a FGO/Ag nanocomposite with promising antibacterial and SERS properties.
\end{abstract}

Keyword: Functionalization; Graphene oxide; Nanocomposites; Silver. 